\title{
Time-Varying Phase Diversity Turbulence Compensation
}

\author{
Adam W.M. van Eekeren, Klamer Schutte, Judith Dijk, Piet B.W. Schwering \\ TNO Defence, Security and Safety, P.O. Box 96864, 2509 JG, The Hague, The Netherlands
}

\begin{abstract}
Long range object identification needs visual identification over large distances. However, atmospheric turbulence does hinder long range imaging. Therefore it is crucial to compensate the visual artifacts due to atmospheric turbulence. In this paper we propose a new method to compensate these turbulence effects, thus enabling identification at larger distances. Our method is based on applying phase diversity imaging by a wavefront modulator in free-running mode. As we have no feedback loop, we can simultaneously compensate turbulence for multiple isoplanatic angles. The wavefront modulator generates several images with known additional wave front aberrations. This extra information allows us to locally estimate the optimal wave front aberration and thus the optimal - turbulence free - image can be derived. This paper provides results on simulated data showing that this method performs well under realistic turbulence and noise conditions. Furthermore the robustness of the proposed method is shown for varying algorithmic settings.
\end{abstract}

Keywords: Turbulence compensation, phase diversity, adaptive optics

\section{INTRODUCTION}

For long range visual observation it is evident that a clear high resolution image is needed. However, imaging over longer ranges is hard as the received power decreases with the square of the distance. In addition, propagation losses decrease the received power with an exponential function. This limits the range over which visual identification is effective. Typically one tries to increase range limitation by increasing the optical aperture of the imaging system. Yet a larger optical aperture results in a system more susceptible to turbulence. Especially in ground-to-ground scenarios the amount of turbulence encountered cannot be ignored. In combination with the need for a large optical aperture it is generally believed that long range visual observation is turbulence-limited. Indeed, turbulence compensation is an enabler for beyond state-of-the-art long range visual observation.

Within astronomy applications turbulence compensation approaches are well known. Except for the Hubble space telescope all high end telescopes are hindered by the Earth's atmosphere. Modern telescopes typically include an adaptive optics part, which corrects the atmospheric distortion. This is enabled by measuring the distortion of a guide star of a laser beam, and correcting that in a real-time loop with adaptive optics. A typical assumption in such a system is that the turbulence effect is constant over the field of view of the telescope. For astronomy applications it is assumed that the turbulence is caused in a single layer, in effect resulting in a single phase screen. However, for ground-to-ground applications the full imaging path will be much closer to the ground. This will result in a more complex turbulence setup. In effect, the isoplanatic angle, i.e. the angle over which the turbulence can be assumed constant, will often be much smaller than the field of view of the camera system. This means that a closed loop adaptive optics system such as used in an astronomy application does not work. Multi-isoplanatic patch systems are conceivable, yet their optical complexity does not scale well with a significant number of phase screens as encountered with ground-to-ground applications.

For long (effective) integration times one can assume that the total effect of turbulence is a constant blur. This assumption is valid when the integration time is so long that it effectively averages all possible turbulence states. In this case the image deterioration by turbulence can be corrected by image deblurring, preferably combined with a procedure where during the longer integration time a correction is made for the tilt leading to image translation. Such procedures ${ }^{1,2}$ prove quite effective in low turbulence scenarios.

Further author information:

E-mail: adam.vaneekeren@tno.nl, Telephone: +31 8886 64047. The presented work in this paper is patent pending under nr. 10158992.7-2217.

Infrared Technology and Applications XXXVII, edited by Bjørn F. Andresen, Gabor F. Fulop, Paul R. Norton, Proc. of SPIE Vol. 8012, 80120D - (C) 2011 SPIE · CCC code: 0277-786X/11/\$18 · doi: 10.1117/12.884580 


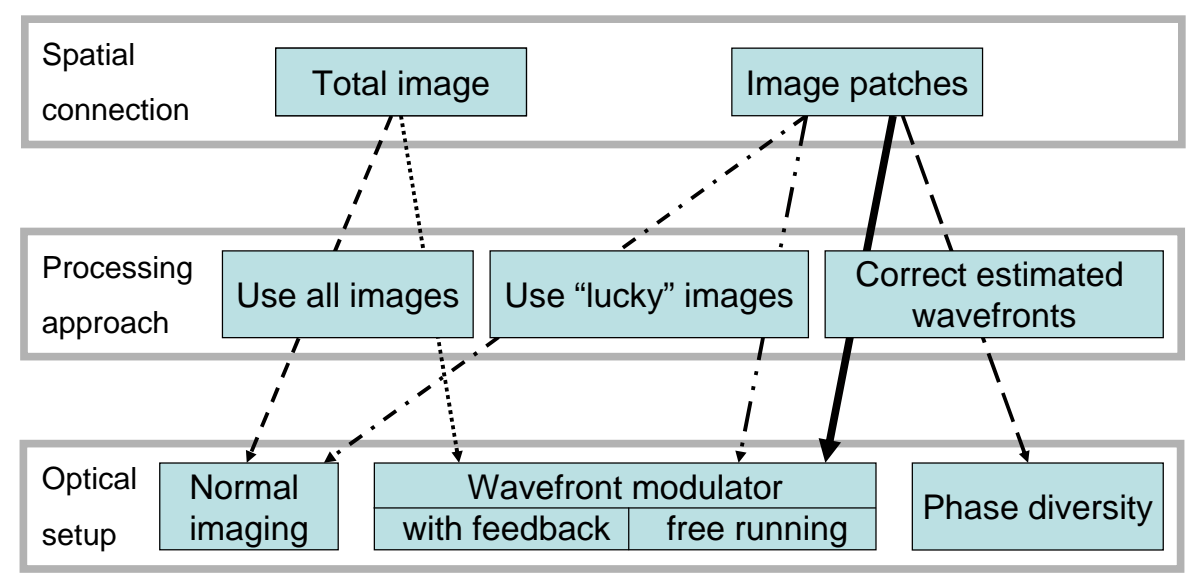

Figure 1. Overview of different turbulence compensation methods. The dashed line represents the methods based on a time-averaged blur. The dotted line represents the successful approach for astronomy applications using adaptive optics. The dot-dash line represents a lucky imaging approach. The long dashes represent the typical phase diversity approach, where the solid line depicts the work discussed in this paper. A variation to our work is represented by the dot-dot-dash line; a lucky imaging approach using a free-running wavefront modulator.

An obvious extension of such an approach is to account the effect of the small isoplanatic angle by applying a similar technique to smaller image patches. ${ }^{3}$ A further step is to acknowledge the time-varying nature of the turbulence by using a lucky imaging technique. ${ }^{4}$ In essence it does acknowledge that in a time series different turbulence distortions are present, and it uses a selection mechanism to select the best image patch in the time series. While lucky imaging approaches can obtain very good results, its downside is the amount of time needed before a sufficient good image patch is seen for every image position. Promising here is the use of phase diversity imaging, ${ }^{5,6}$ which significantly improves the time to observe good image patches.

Ordinary imaging is only capable of measuring the power distribution of an image. Phase diversity however does record not a single but several (often two) images simultaneously. These different images differ by a known phase abberation, such as a defocus. Having multiple images allows to not only reconstruct the power of the image but also its phase components. In turn, also knowing its phase, allows to truly correct any distortion, where the earlier discussed long-exposure approaches typically resort to a phase-free blind deconvolution deblur approach. Similar to such deblurring approaches the phase diversity approach can be applied to non-isoplanatic imaging conditions. ${ }^{7,8}$

Phase diversity imaging needs a setup where the optical path is typically split in an in-focus and a diversity image. In Section 4 results are presented which show that the correction achievable by phase diversity is best, close to the actual distortion, and that reconstructions for points further off in the abberation-configuration space are less accurate. For this reason we propose a setup consisting of a single optical path, where the phase diversity is obtained using a wavefront modulator in free-running mode. This wavefront modulator is rapidly sweeping through the total abberation-configuration space. When its scan rate is higher than the actual change rate of the atmospheric turbulence this guarantees that we obtain images with only very small residual errors. In addition, we know the changes in time of the wavefront modulator such that we can apply phase diversity.

Section 2 describes the used turbulence and imaging model, where Section 3 describes the proposed method for turbulence compensation. In Section 4 results are given, showing the robustness of this approach against initial configuration error, noise, time drift and model assumptions. Finally, Section 5 summarizes these results and provides an outlook to future applications.

\section{TURBULENCE AND IMAGING MODEL}

This section describes the forward model to construct a camera frame, degraded by atmospheric turbulence, from a noise-free and optimal representation of the captured scene. 


\subsection{Turbulence model}

Atmospheric turbulence is caused by spatial-varying and time-varying optical properties of the atmosphere. Assuming a high frame rate camera, atmospheric turbulence can locally, for one isoplanatic angle, be modeled at the image plane with a point spread function (PSF). In this subsection we will explain the relationship between turbulence and the PSF.

Let us assume that the wavefront at the aperture of the camera is defined as:

$$
W(x, y)=A(x, y) e^{i \phi(x, y)}
$$

with $A(x, y)$ the amplitude and $\phi(x, y)$ the phase distribution along the aperture and $(x, y)$ normalized coordinates. Note that the wavefront does not contain information about the scene, but only information about turbulence effects and focusing of the lenses. In this paper we assume that the lenses are perfect in focus, so the wavefront contains only information about turbulence effects. The phase distribution can be expressed as a polynomial:

$$
\phi(x, y)=c_{0}+c_{x 1} x+c_{y 1} y+c_{x 2} x^{2}+c_{y 2} y^{2}+\ldots
$$

The linear terms $\left(c_{x 1}, c_{y 1}\right)$ in this equation cause a horizontal or a vertical shift of the image and the quadratic terms $\left(c_{x 2}, c_{y 2}\right)$ cause a defocus of the image. In this paper we will focus on the quadratic terms.

From the wavefront in (1) a PSF can be calculated with: ${ }^{9}$

$$
\operatorname{PSF}\left(f_{x}, f_{y}\right)=|F[W(x, y)]|^{2}
$$

where $F[$.$] is the Fourier transformation and f_{x}, f_{y}$ are the horizontal and vertical spatial frequencies. Each spatial frequency corresponds with an absolute coordinate $\left(x_{a}, y_{a}\right)$ in the image plane with the optical axis as origin:

$$
\left(x_{a}, y_{a}\right)=\frac{b \lambda}{2 r}\left(f_{x}, f_{y}\right),
$$

where $b$ is the distance from the lens to the image plane, $\lambda$ is the wavelength and $r$ is the aperture radius. Given the pixel pitch $p$ of the sensor and the absolute coordinates it is fairly easy to extract a discrete PSF:

$$
P S F[m, n]=P S F\left(x_{a}, y_{a}\right) \cdot \sum_{m=-K}^{m=K} \sum_{n=-K}^{n=K} \delta\left(x_{a}-m p, y_{a}-n p\right)
$$

In this paper a discrete PSF is used of $9 \times 9$ pixels $(K=4)$.

\subsection{Imaging model}

We model a camera's field-of-view - the scene - at time $t$ as a continuous function $z_{t}$ without degradation due to motion, blur or noise (see Figure 2).

First this continuous representation of the scene is propagating through the atmosphere. Let us define the scene after atmospheric propagation as:

$$
u_{t}=P S F_{t}(\vec{c}) * z_{t}
$$

Here, $P S F_{t}$ indicates a continuous PSF and the vector $\vec{c}=\left[c_{0}, c_{x 1}, c_{y 1}, c_{x 2}, c_{y 2}, \ldots\right]$ denotes a set of polynomial coefficients as used in (2). Now assume that we have adaptive optics with which we can modulate the phase distribution of the wavefront. Given a modulation $\vec{\Delta}_{i}$ the modulated image is defined as: 


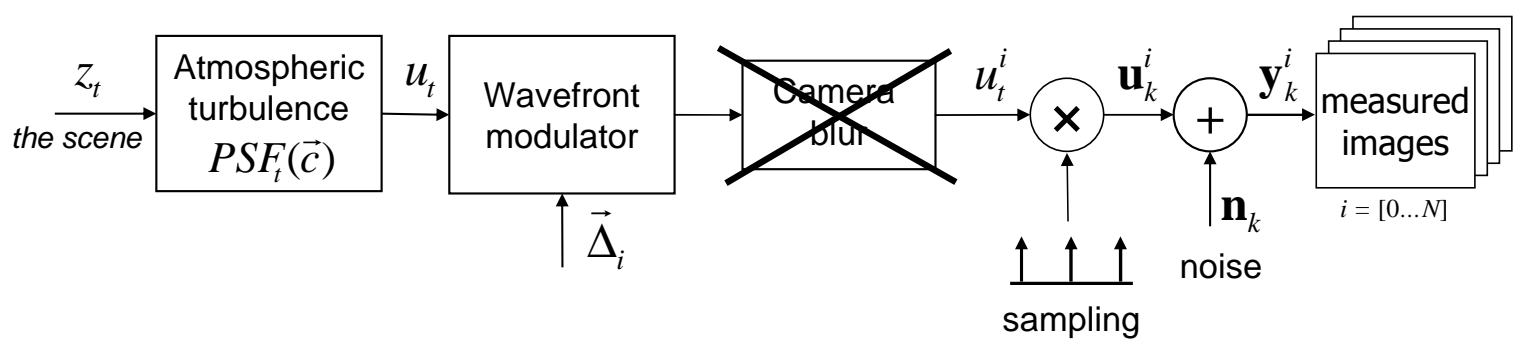

Figure 2. Flow diagram illustrating the degradation of a continuous scene $z_{t}$ into a turbulence deteriorated and modulated images $\mathbf{y}_{k}^{i}$. Note that no camera blur is modeled because we assume that the lenses are perfect in focus.

$$
u_{t}^{i}=P S F_{t}\left(\vec{c}+\vec{\Delta}_{i}\right) * z_{t} .
$$

We will refer to this modulation of the phase distribution as 'phase diversity'. An image without additional phase diversity $\left(\vec{\Delta}_{0}=\mathbf{0}\right)$ is denoted with $u_{t}^{0}$ and is equal to $u_{t}$. Note that no camera blur is modeled because we assume that the lenses are perfect in focus. However, if camera blur will be present, it will be compensated by modeling it as part of the atmospheric turbulence.

Next the continuous, deteriorated and modulated image is sampled by the camera sensor and noise is added. The noise is modeled by additive, independent and identically distributed Gaussian noise samples $\mathbf{n}_{k}$ with standard deviation $\sigma_{n}$. The final measured images are defined as:

$$
\mathbf{y}_{k}^{i}[m, n]=u_{t}^{i}\left(x_{a}, y_{a}\right) \cdot \sum_{m=1}^{m=W} \sum_{n=1}^{n=H} \delta\left(x_{a}-m p, y_{a}-n p\right)+\mathbf{n}_{k},
$$

with $\left(x_{a}, y_{a}\right)$ the absolute coordinates in the image plane and $p$ the pixel pitch of the sensor.

For practical purposes we modeled the scene in our experiments not as a continuous function, but as a discrete image $\mathbf{z}_{k}$. In other words, we put the 'sampling'-step in Figure 2 at the beginning of the flow diagram. This leads to the following definition:

$$
\mathbf{y}_{k}^{i}=P S F_{k}\left(\vec{c}+\vec{\Delta}_{i}\right) * \mathbf{z}_{k}+\mathbf{n}_{k},
$$

with $\mathbf{z}_{k}$ the discrete representation of the scene before degradation.

\section{METHOD}

In this section the minimization method will be described to estimate an optimal image from a turbulence deteriorated image of a scene and some 'phase modulated images'. To find such an optimal image, we solve an inverse problem based on the imaging model described in the previous section. The cost function that we iteratively minimize is defined as:

$$
C\left(\hat{\mathbf{z}}_{k, q}, \vec{c}_{e s t, q}\right)=\sum_{i=0}^{N} \sum_{m, n}^{W, H}\left(P S F_{k}\left(\vec{c}_{e s t, q}+\vec{\Delta}_{i}\right) * \hat{\mathbf{z}}_{k, q}-\mathbf{y}_{k}^{i}\right)^{2},
$$

with $q$ the $q$-th iteration of the minimization procedure and $[m, n]$ the pixel indices in respectively horizontal and vertical direction. A flow diagram of the minimization is depicted in Figure 3.

One iteration of our minimization procedure consists of two steps: 1) finding an optimal image $\hat{\mathbf{z}}_{k, q}$ that minimizes $C$ while $\vec{c}_{e s t, q}$ is fixed and 2) finding the optimal $\vec{c}_{e s t, q}$ that minimizes $C$ while keeping $\hat{\mathbf{z}}_{k, q}$ fixed. The minimization procedure is split into two steps because minimizing the cost function for both the image and the wavefront phase coefficients at the same time would lead to a complex and very slow convergence schema. 


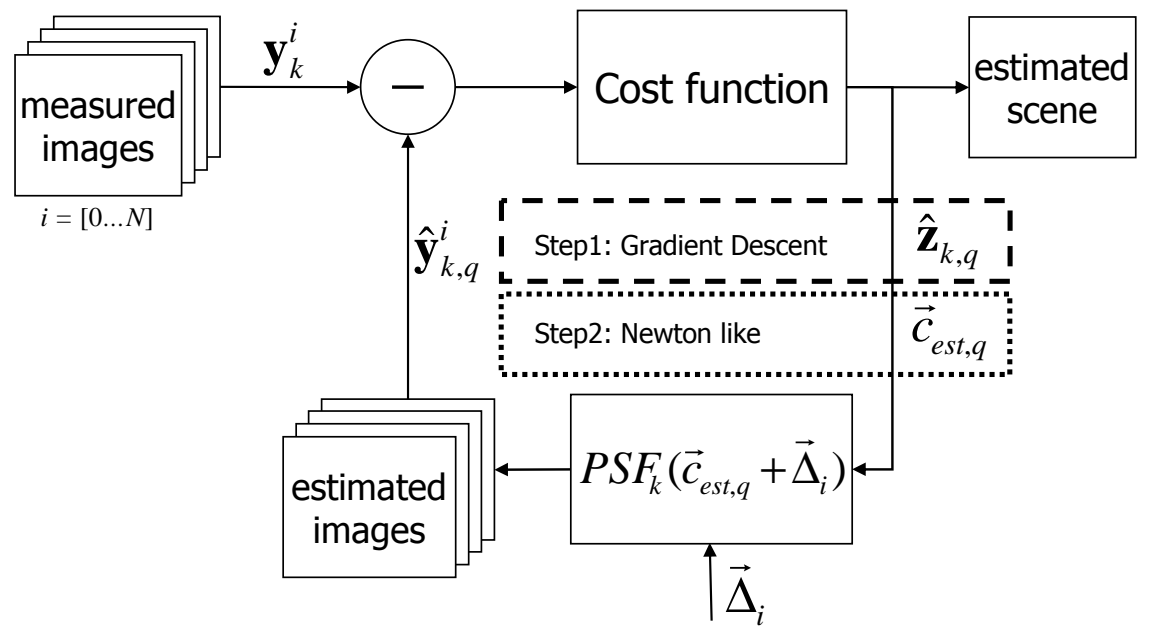

Figure 3. Flow diagram illustrating the minimization procedure in order to estimate the original scene $\mathbf{z}_{k}$ and the wavefront phase coefficients $\vec{c}$ caused by the turbulence. $N$ is the number of measured images used in the minimization and $q$ indicates the $q$-th iteration of the minimization. Each minimization cycle is performed in two steps: first the scene $\mathbf{z}_{k}$ is estimated using a Gradient Descent approach, next the wavefront phase coefficients $\vec{c}$ are estimated using a Newton like approach.

The first step is performed with a Gradient Descent optimization as described in Hardie et al. ${ }^{10}$ The optimization of the wavefront coefficients $\vec{c}_{e s t, q}$ is done with a subspace trust-region method, which is based on the interior-reflective Newton method. ${ }^{11,12}$

\section{EXPERIMENTAL RESULTS}

This section shows experimental results on simulated data, which has the advantage that the obtained results can be compared with a ground truth. Furthermore, in such a controlled environment it is easy to verify the influence of e.g. noise, convergence behavior and modeling errors.

\subsection{Image containing varying defocus}

As a first experiment we show the performance of our method on a simulated image $(64 \times 64$ pixels $)$ with a realistic Signal-to-Noise Ratio $(\mathrm{SNR}=30 \mathrm{~dB}$ ) containing a varying defocus (see Figure 4 (a)). Here, SNR is defined as $\left.20 \log _{10}\left(\left(\max \left(\mathbf{y}_{k}\right)-\min \left(\mathbf{y}_{k}\right)\right) / \operatorname{std}\left(\mathbf{n}_{k}\right)\right)\right)$. The defocus increases from left to right, with on the left outside of the image little defocus $\left(c_{x 2}=5\right)$ and on the right outside much defocus $\left(c_{x 2}=30\right)$. The defocus in vertical direction is constant $\left(c_{y 2}=5\right)$. Processing was done on tiles of $16 \times 16$ pixels and we used three different 'phase modulated images' $\left(\vec{\Delta}_{1}=[10,17], \vec{\Delta}_{2}=[-20,0], \vec{\Delta}_{3}=[10,-17]\right)$ in our minimization procedure (see Figure 4 (b)-(d)). The resulting image is depicted in (f). This result is very close to the ground truth image (e), which is also depicted by the residue image in $(\mathrm{g})$.

\subsection{Convergence behavior of defocus parameters}

An important step in our method is the selection of the 'phase modulated images'. Therefore we set up two experiments to test the effect of different 'phase modulated images' on the estimated result. As input image we simulated a noise free image with defocus parameters $c_{x 2}=c_{y 2}=20$ (see Figure 5). This input image, which has a residue value (mean squared error per pixel) of 0.0092 with the ground truth, was modulated with three different phase diversities as depicted at the bottom-left part of Figure 5. One set of modulations can be described with an amplitude $A$ and angle $\phi$. At the right part of Figure 5 the residue value is plotted against $\phi$ for five different amplitudes. Small residue values are obtained for amplitudes $(A=10, A=20, A=30)$ which are close to the defocus value of the input image. 


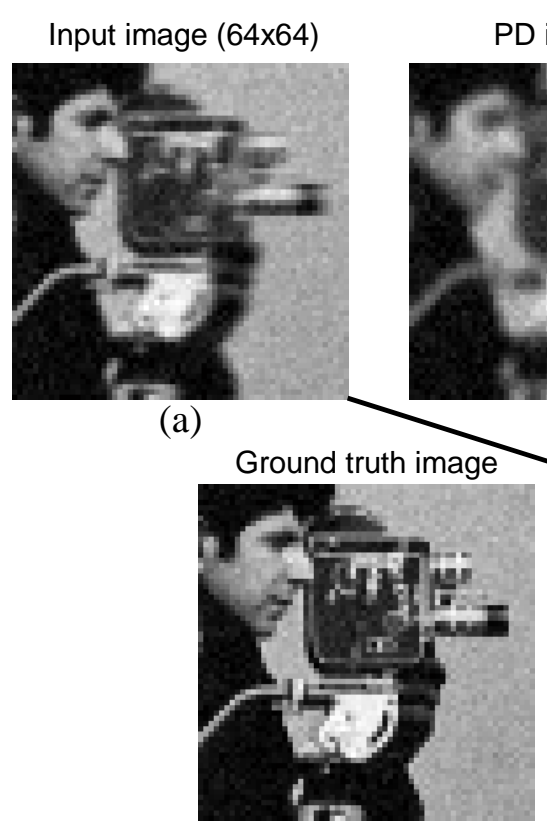

(e)
PD image 1

(b)

Estimated image

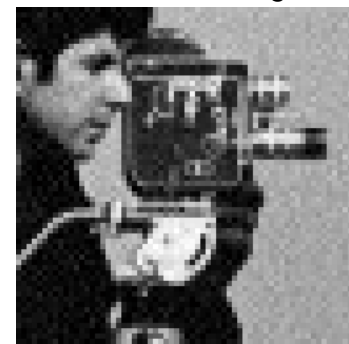

(f)
PD image 2

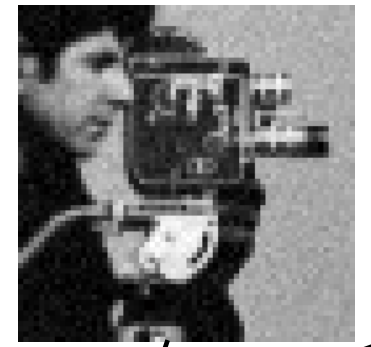

(c)

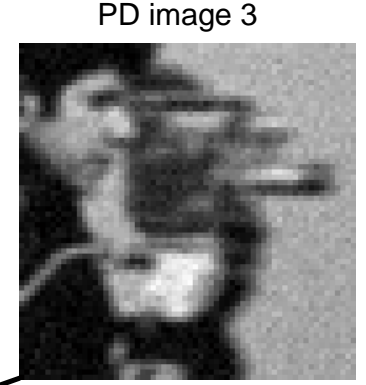

(d)

Figure 4. (a) Input image $[0,1]$ with spatially varying defocus (increasing from left to right), (b)-(d) Three different phase-diversity images, (e) Ground truth image, (f) Estimated image after turbulence compensation with the proposed method, (g) Residue [-0.5, 0.5] between (e) and (f).
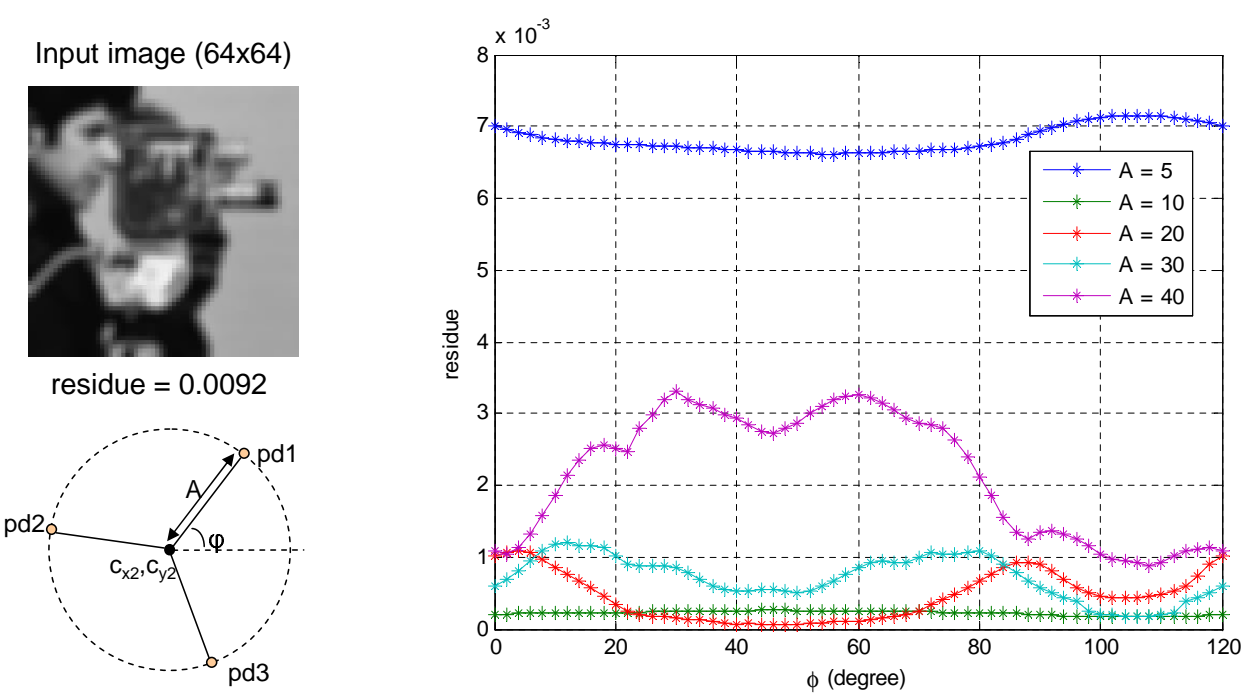

Figure 5. Residue between estimated results and ground truth for different sets of phase modulated images. One set of phase modulated images is described by an amplitude $A$ and an angle $\phi$.

A second experiment was done where the angle $\phi$ was fixed at 60 degrees. For varying amplitudes $A$ and different amount of defocus $c_{x 2}=c_{y 2}$ the residue is calculated and depicted in Figure 6. From this figure it can be deduced that an amplitude which is close to the amount of defocus in the input image results in the lowest residue value. It also shows that the more defocused the input image is, the more difficult it is to estimate an image which is close to the ground truth image. 


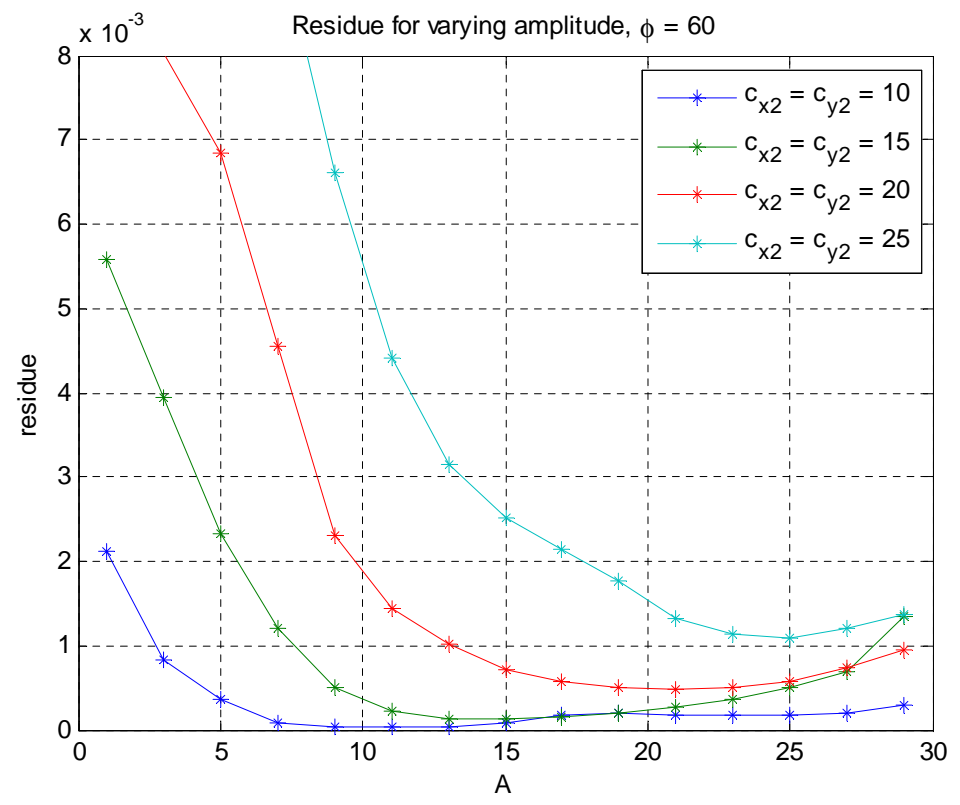

Figure 6. Residue between estimated results and ground truth for different sets of phase modulated images (varying amplitude). Each curve represents a certain amount of defocus $c_{x 2}=c_{y 2}$ of the input image.

\subsection{Variation of defocus parameters}

Due to the time-varying nature of turbulence there will be a small difference between the assumed and real defocus of the phase-diversity images. To test this influence we conducted an experiment where we added normally distributed noise to the used phase diversities $\left(\vec{\Delta}_{1}=[10,17], \vec{\Delta}_{2}=[-20,0], \vec{\Delta}_{3}=[10,-17]\right)$. For each amount of noise 100 realizations are processed. As input image we used a noise free image with defocus parameters $c_{x 2}=c_{y 2}=20$ (see Figure 5). The plot in Figure 7 depicts the influence of phase diversity variation on the residue value. The stars indicate the mean residue value and the vertical lines indicate two times the standard variation given 100 measurements. It shows that our method can handle a fair amount of variation between the actual and assumed phase diversity. Note that for large variation between the actual and assumed phase diversity, the variation in the estimated result is large as well.

\subsection{Exact vs approximate model}

To validate the robustness of our method to modeling errors, an experiment is performed in which we processed an image with varying defocus (a noise-free version of the image in Figure 4 (a)) in two different ways. First we processed the image with exactly the same model as used to create it and next we processed the same image using an approximate model. The approximate model differs from the original model because the PSF in (3) is calculated on a 16 times less dense grid. This makes the used PSF (5) less accurate than the one used in the original model. At the right-side of Figure 8 the difference is visible in the cross-section of a PSF kernel created with both models $\left(c_{x 2}=c_{y 2}=20\right)$. From Figure 8 it is clear that the residue values of both results are small and that the results are visually very similar.

\section{DISCUSSION}

From the experiments the following conclusions can be drawn:

- The proposed method is able to cope with spatial varying blur under a realistic Signal-to-Noise Ratio. 


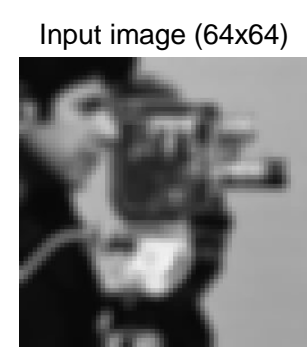

residue $=0.0092$

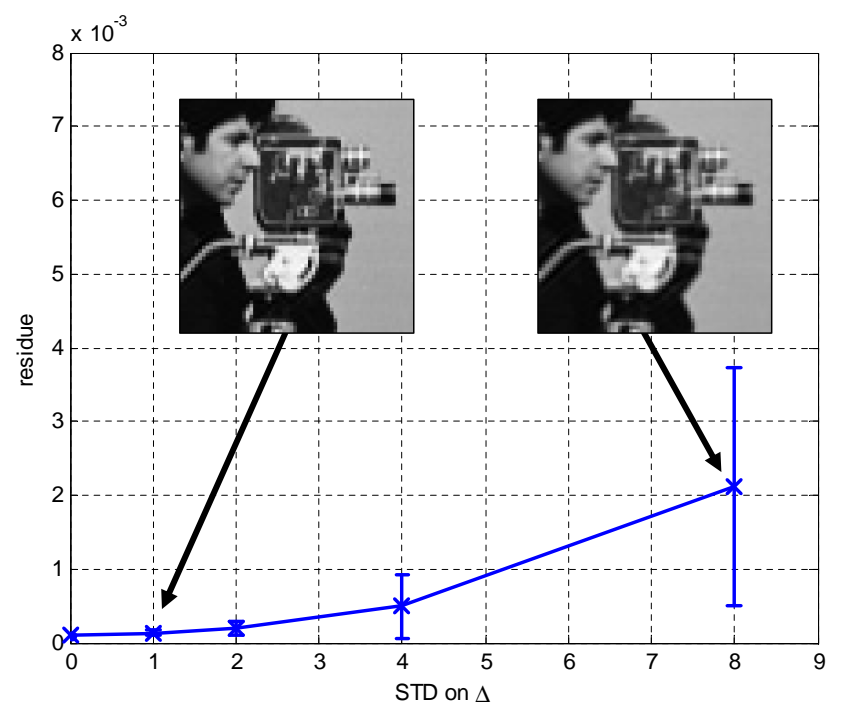

Figure 7. Residue between estimated results and ground truth for variation between the actual and assumed phase diversity.

Input image $(64 \times 64)$

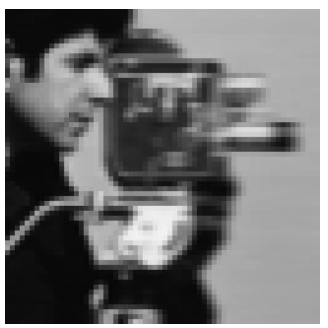

residue $=3.9 \times 10^{-3}$
Estimated image (exact model)

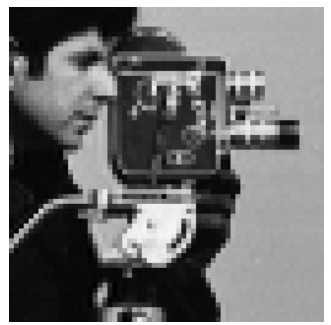

residue $=1.7 \times 10^{-4}$
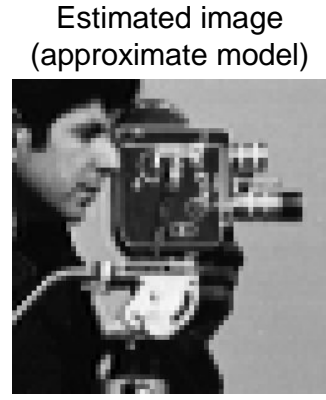

residue $=2.1 \times 10^{-4}$
PSF kernel exact vs. approximate

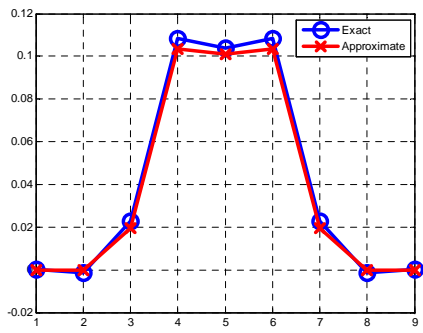

Figure 8. Left: input image [0,1], middle-left: estimated image using an exact model, middle-right: estimated image using an approximate model. Residue values indicate the mean squared error between the estimated results and the ground truth. Right: cross-section of PSF kernel exact model vs. approximate model for $c_{x 2}=c_{y 2}=20$.

- For a phase diversity turbulence compensation approach one should limit the difference between the closest recorded and turbulence-free image. This means that a time-varying approach using a wavefront modulator is preferable to a static double imaging setup.

- The algorithm can cope with limited differences in wavefronts as will be the result of time-variation of turbulence.

Next step in development shall be verification of this system concept with a hardware demonstrator against real turbulence. Especially the current assumption that we can sufficiently fast (in comparison to actual temporal turbulence changes) sample the phase diversity configuration space should be proved in practice. High camera frame rates will definitely aid, possibly in a frame-burst mode to circumvent read-out bottlenecks.

Assuming a positive outcome of the real-world tests, the results so far make us expect that a camera system applied with time-varying phase diversity turbulence compensation will outperform any other known system concept for long-range ground-to-ground imaging. 


\section{REFERENCES}

[1] Schwering, P., van den Broek, S., and van Iersel, M., "Eo system concepts in the littoral," SPIE Proc., Infrared Technology and Applications XXXIII 6542 (2007).

[2] Huebner, C. S. and Greco, M., "Blind deconvolution algorithms for the restoration of atmospherically degraded imagery: a comparative analysis," SPIE Proc., Optics in Atmospheric Propagation and Adaptive Systems XI $\mathbf{7 1 0 8}$ (2008).

[3] Huebner, C. S., "Compensating image degradation due to atmospheric turbulence in anisoplanatic conditions," SPIE Proc., Mobile Multimedia/Image Processing, Security, and Applications 7351 (2009).

[4] Woods, S. C., Burnett, J. G., Kent, P. J., and Turner, A. J., "High-resolution imaging using lucky frame selection," Defence Codex, The magazine for Defence Engineering and Science 2, 1-12 (2008).

[5] Gonsalves, R. A., "Phase retrieval and diversity in adaptive optics," Optical Engineering 21(5), 829-832 (1982).

[6] Korkiakoski, V., Keller, C., Doelman, N., Fraanje, R., and Verhaegen, M., "Joint-optimization of phasediversity and adaptive optics," Adaptive Optics: Methods, Analysis and Applications (AO), OSA Topical Meeting (2011).

[7] Gonsalves, R. A., "Nonisoplanatic imaging by phase diversity," Optics Letters 19(7), 493-495 (1994).

[8] Paxman, R. G., Thelen, B. J., and Seldin, J. H., "Phase-diversity correction of turbulence-induced spacevariant blur," Optics Letters 19(16), 1231-1233 (1994).

[9] Luke, D. R., Burke, J. V., and Lyon, R. G., "Optical wavefront reconstruction: Theory and numerical methods," SIAM Review 14(2), 169-224 (2002).

[10] Hardie, R. C., Barnard, K. J., Bognar, J. G., Armstrong, E. E., and Watson, E. A., "High-resolution image reconstruction from a sequence of rotated and translated frames and its application to an infrared imaging system," Optical Engineering 37(1), 247-260 (1998).

[11] Coleman, T. and Li, Y., "On the convergence of reflective newton methods for large-scale nonlinear minimization subject to bounds," Mathematical Programming 67(2), 189-224 (1994).

[12] Coleman, T. and Li, Y., "An interior, trust region approach for nonlinear minimization subject to bounds," SIAM Journal on Optimization 6, 418-445 (1996). 